\title{
Self-induction of abortion among women accessing second- trimester abortion services in the public sector, Western Cape Province, South Africa: An exploratory study
}

\author{
D Constant, ${ }^{1} \mathrm{MPH}, \mathrm{MSc}$ (Anat); D Grossman, ${ }^{2} \mathrm{MD} ; \mathrm{N}$ Lince, ${ }^{3} \mathrm{MPH}, \mathrm{MIA} ; \mathrm{J}$ Harries, ${ }^{1} \mathrm{PhD}, \mathrm{MPH}$ \\ ${ }^{1}$ Women's Health Research Unit, School of Public Health and Family Medicine, University of Cape Town, Cape Town, South Africa \\ ${ }^{2}$ Ibis Reproductive Health, Oakland, CA, and Bixby Center for Global Reproductive Health and San Francisco General Hospital, \\ Department of Obstetrics, Gynecology and Reproductive Sciences, University of California, San Francisco, CA, USA \\ ${ }^{3}$ Ibis Reproductive Health, Johannesburg, South Africa (current affiliation: Health Economics and Epidemiology Research Office, \\ Department of Internal Medicine, Faculty of Health Sciences, University of the Witwatersrand, Johannesburg, South Africa)
}

Corresponding author: D Constant (deborah.constant@uct.ac.za)

\begin{abstract}
Background. Despite South Africa's liberal abortion law permitting abortion on request in the first trimester and under restricted conditions for second-trimester pregnancies, the practice of unsafe self-induced abortion persists. However, the prevalence of this practice, the methods used and the reasons behind it are relatively under-researched. As part of a larger study seeking to improve abortion services in the Western Cape Province, we explored reports of prior attempts to self-induce abortion among women undergoing legal second-trimester abortion. Objectives. To describe the prevalence and methods of and factors related to unsuccessful attempts at self-induction of abortion by women presenting without complications and seeking second-trimester abortion at public health facilities in the Western Cape.

Methods. In a cross-sectional study from April to August 2010, 194 consenting women undergoing second-trimester abortion were interviewed by trained fieldworkers using structured questionnaires at four public sector facilities near Cape Town.

Results. Thirty-four women (17.5\%; 95\% confidence interval 12.7 - 23.4) reported an unsuccessful attempt to self-induce abortion during the current pregnancy before going to a facility for second-trimester abortion. No factors were significantly associated with self-induction, but a relatively high proportion of this small sample were unemployed and spoke an indigenous African language at home. A readily available herbal product called Stametta was most commonly used; other methods included taking tablets bought from unlicensed providers and using other herbal remedies. No use of physical methods was reported.

Conclusions. The prevalence of unsafe self-induction of abortion is relatively high in the Western Cape. Efforts to inform women in the community about the availability of free services in the public sector and to educate them about the dangers of self-induction and unsafe providers should be strengthened to help address this public health issue.
\end{abstract}

S Afr Med J 2014;104(4):302-305. DOI:10.7196/SAMJ.7408

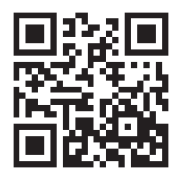

From 1994 to 2005, the proportion of materna mortality attributable to unsafe abortion in South Africa (SA) decreased by an estimated $91 \% .^{[1]}$ This reduction was attributed to the legalisation of abortion in 1996 with the passing of the Choice on Termination of Pregnancy Act, which provided for abortion on demand through 12 weeks of gestation and for several conditions, including socioeconomic hardship, through 20 weeks of gestation.

Self-induced abortion involves the use of medications other than evidence-based medical abortion regimens, as well as other substances or physical trauma, to try to end an unwanted pregnancy. Selfinduction is synonymous with unsafe abortion, defined by the World Health Organization (WHO) as 'a procedure for termination of an unintended pregnancy done either by people lacking the necessary skills or in an environment that does not conform to minimum medical standards or both. ${ }^{[2]}$ Conditions defining unsafe abortion practices include some or all of the following: absence of pre-abortion counselling, induction by an unskilled provider, procedures undertaken in unhygienic conditions, ingestion of traditional medication or hazardous substances, use of physical devices, or incorrect prescription of medication with inadequate instructions for use and no follow-up. ${ }^{[2]}$

In developed countries with liberal abortion laws, unsafe abortion is generally infrequent, while in developing countries, where access is often restricted, as many as $77 \%$ of all induced abortions may be unsafe. ${ }^{[2]}$ The Guttmacher Institute and the WHO estimate that $58 \%$ of abortions were unsafe in 2008 in the southern African region, which includes countries beyond $\mathrm{SA},{ }^{[3]}$ and this figure is supported by other country-specific studies. In the 2nd South African national Youth Risk Behaviour Survey, ${ }^{[4]} 6 \%$ of female high-school learners reported having had an abortion, and $9.8 \%$ of male learners reported that their female partners had done so. Of these, only $51.5 \%$ reported that the abortion had been performed at a hospital or clinic; $20.5 \%$ of respondents had consulted a traditional healer, $10.2 \%$ went to 'another place', and for $5.4 \%$ the provider setting was unknown. Neither age nor race was associated with differences in where the abortion took place, but there were associations between seeking help from a traditional healer and both earlier school grade and geographical region. The highest prevalence of using a traditional healer was in KwaZulu-Natal.

Data from the USA have shown that self-induction was associated with a delay in seeking or obtaining a clinic-based abortion. ${ }^{[5]}$ Previous research has reported that adult SA women sought abortion care outside designated facilities because of perceived poor quality of services, oversubscribed services, ineffective referral systems within health services, and lack of knowledge of the time-limited nature of the service. ${ }^{[6]}$ More recent research in SA has identified additional barriers to safe abortion, such as uneven provision between rural 
and urban areas, unsubstantiated rumours around potential harms related to abortion, and intimidation by and negative attitudes from pre-abortion counsellors. ${ }^{[7.8]}$

Grossman et al. ${ }^{[9]}$ found that delays in accessing services were common among women seeking second-trimester abortion services in the Western Cape, SA. We hypothesised that these delays may have been associated with prior attempts to self-induce, and undertook this exploratory study to assess this question. We describe the prevalence and practices of unsuccessful attempts at self-induction and related factors among women presenting without complications and seeking secondtrimester abortion at public health facilities in the Western Cape.

\section{Methods}

This study forms part of a larger, repeated cross-sectional study of women undergoing second-trimester abortion. The baseline survey was completed in 2008, ${ }^{[9]}$ and the data reported here are derived from a second round of data collection in 2010. Study sites included four public hospitals in the Western Cape that provided second-trimester abortion services, which were selected according to the volume of women seeking abortion services. Some facilities provided surgical abortion (dilation and evacuation), while others provided medical induction using misoprostol. Women were referred to these facilities based on proximity to their home. Three of the four sites served urban communities in the Cape Town metropolitan area, while the fourth served a small town roughly $120 \mathrm{~km}$ away in a primarily agricultural area.

Ethical approval was obtained from the University of Cape Town Human Research Ethics Committee, the Stellenbosch University Committee for Human Research, and the Allendale Investigational Review Board. All participants provided written informed consent, and confidentiality and anonymity were ensured.

The study was conducted between April and August 2010. All women attending the hospitals for second-trimester abortion on days when study interviewers were present were invited to participate. Eligibility criteria included age 18 years or older, gestational age between 12.1 weeks and 20.9 weeks, and ability to communicate in English or Xhosa. Bilingual interviewers administered questionnaires in a private location at the hospitals after the women had recovered from the abortion. Data were obtained on demographic and socioeconomic indicators, reproductive history and care-seeking behaviour. In addition, participants were asked whether they had tried any other methods to end the pregnancy before the abortion procedure, and if so, what method they had attempted. Qualitative, open-ended responses were recorded and later coded into categories.

Data were analysed using Stata (version 12, College Station, TX, USA). A priori factors identified for potential association with selfinduction included age, education, socioeconomic status, home language, parity, previous abortions, gestational age according to ultrasound scan, and care-seeking experiences. Proportions were compared using $\chi^{2}$ tests or Fisher's exact tests when appropriate. When means were compared, Students $t$-tests were used. Two-sided significance tests were used throughout, and valid percentages are reported for all results. The number of subjects recruited was sufficient to detect a prevalence of self-induction of $20 \%( \pm 10 \%)$, with $90 \%$ power and a two-sided alpha of 0.05 .

\section{Results}

At the four facilities involved in the study, a total of 698 women had second-trimester abortions performed during the sampling period. For logistical reasons, we were only able to obtain consent from and interview $28.1 \%$ of them $(N=196)$. Two interviews were excluded because the forms were largely incomplete, so the final sample consisted of questionnaires from 194 women.
A total of $34 / 194(17.5 \%$; $95 \%$ confidence interval 12.7 - 23.4) women interviewed reported that they had attempted to end their current pregnancy before coming to the facility. Table 1 compares characteristics of the women who had and had not attempted prior selfinduction. Means, medians and standard deviations, or percentages by self-induction (yes/no), are shown for factors that were hypothesised a priori potentially to be associated with self-induction. These factors included age, educational level achieved, home language, type of housing and employment status (as a proxy for socioeconomic status), parity, prior abortion, gestational age at first ultrasound examination (usually at a different facility), and gestational age as calculated on the day of admission for the abortion procedure. In this small exploratory sample, the results suggest that self-induction was most common among unemployed women and those speaking an indigenous African language at home; however, no significant associations with any of these factors emerged. There also appeared to be a trend towards presenting at a later gestational age at the time of first ultrasound among women who had attempted self-induction compared with those who had not, but this association was also not significant.

Table 2 summarises the methods women used for self-induction. In 2 of the 34 cases the method used for self-induction was not recorded. The most common practice (17/32, 53.1\%) was ingestion of an herbal product called Stametta. According to the manufacturer's details listed on the package, Stametta can be used for a wide range of ailments such as painful menstruation and fatigue, and to improve general well-being. The product label cautions that it should not be used by pregnant women and children under the age of 14 years. The composition includes a blend of aloe, ascorbic acid, aniseed oil, magnesium sulphate and preservative. Stametta is readily available in drug stores and shops. Other methods women used included responding to an advertisement by an illegal backstreet provider and taking tablets (unnamed) (5/32, 15.6\%), using other herbal remedies $(6 / 32,18.7 \%)$, using other medications such as emergency contraceptives or antibiotics $(2 / 32,6.2 \%)$, or 'smoking a lot', probably cigarettes $(1 / 32,3.1 \%)$.

Two women elaborated on their experience and their reasons for attempting self-induction. One woman who consulted an illegal backstreet provider said that she was given three tablets to take at home and that the provider called twice, but then disappeared. The other respondent explained that she had been turned away from a healthcare facility because she was there too late in the day to be seen. She then called a number from an advertisement and paid R1 600 (approximately US\$220) for tablets which she took, but did not abort.

\section{Discussion}

Despite liberal abortion laws and previous reductions in maternal mortality attributable to unsafe abortion, SA continues to face problems with unsafe abortion and a relatively high rate of self-induction. SA's Confidential Enquiry on Maternal Death (CEMDSA) or 'Saving Mothers' reports for $2002-2004^{[10]}$ and $2005-2007^{[11]}$ showed that unsafe abortion contributed to $3.5 \%$ and $3.4 \%$ of all maternal mortality, respectively. However, in the 2005 - 2007 report, this proportion rose to $4.9 \%$ after the figures for misclassified HIV/AIDS-related deaths were adjusted ${ }^{[11]}$ Unfortunately, in the latest report, for 2008 - 2010, ${ }^{[12]}$ the CEMDSA committee reclassified abortion-related deaths as 'unsafe miscarriage', so the reports are not directly comparable; however, the prevalence was $3.8 \%$ for this period.

The prevalence of self-induced abortion (17.5\%) reported in this study is high compared with developed countries where abortion is legal. In two recent US studies, self-induction was reported by $4.6 \%$ of everpregnant women ${ }^{[5]}$ and by $2.6 \%$ of women attending abortion clinics. ${ }^{[13]}$

Although not significant, the results from our exploratory study show that self-induction was more common among African language- 
Table 1. Key characteristics of patients seeking second-trimester abortion and reporting previous attempts to self-induce

\begin{tabular}{|c|c|c|c|}
\hline Characteristic & $\begin{array}{l}\text { Attempted self-induction } \\
(N=34)\end{array}$ & $\begin{array}{l}\text { Did not attempt self-induction } \\
(N=160)\end{array}$ & $p$-value ${ }^{*}$ \\
\hline Age (years), mean (median) $\pm \mathrm{SD}$ & $25.7(23.5) \pm 5.50$ & $26.5(26) \pm 5.65$ & 0.358 \\
\hline School education (grade), $n(\%)$ & & & 0.600 \\
\hline$<9$ & $1(2.9)$ & $8(5.0)$ & \\
\hline $9-12$ & $33(97.1)$ & $151(95)$ & \\
\hline Home language: Xhosa/Zulu/Tswana, $n(\%)$ & $29(85.3)$ & $122(76.3)$ & 0.249 \\
\hline SES: Informal housing, $n(\%)$ & $24(70.6)$ & $103(64.4)$ & 0.489 \\
\hline SES: No paid work, $n(\%)$ & $25(73.5)$ & $96(60.0)$ & 0.139 \\
\hline Parity, mean (median) $\pm \mathrm{SD}$ & $\begin{array}{l}N=33 \\
1.1(1) \pm 0.96\end{array}$ & $\begin{array}{l}N=153 \\
1.3(1) \pm 1.03\end{array}$ & 0.409 \\
\hline Pregnancies (incl. this pregnancy), mean (median) $\pm \mathrm{SD}$ & $2.2(2) \pm 0.99$ & $2.4(2) \pm 1.15$ & 0.570 \\
\hline Had a prior TOP, $n(\%)$ & $1(2.9)$ & $6(3.8)$ & 0.645 \\
\hline Gestational age at first US (weeks), $n(\%)$ & & & 0.116 \\
\hline Mean (median) \pm SD & $14.9(14.7) \pm 2.78$ & $14.5(14) \pm 2.14$ & 0.322 \\
\hline$<16$ & $21(61.8)$ & $120(75)$ & \\
\hline $16.1-19.9$ & $13(38.2)$ & $40(25)$ & \\
\hline Gestational age at admission (weeks), mean (median) \pm SD & $16.0(15.6) \pm 2.09$ & $15.8(15.6) \pm 1.94$ & 0.569 \\
\hline
\end{tabular}

Table 2. Methods used for attempted self-induction of abortion

\begin{tabular}{ll}
\hline Method used & $\begin{array}{l}\boldsymbol{N}=\mathbf{3 2} \\
\boldsymbol{n}(\%)\end{array}$ \\
\hline Back-street abortion provider & $6(18.7)$ \\
$\quad$ Saw advertisement - tablet taken & $5(15.6)$ \\
Saw advertisement - only called, didn't go & $1(3.1)$ \\
Self-medication method & $26(81.2)$ \\
Stametta & $17(53.1)$ \\
Other remedies - aloe, lemon, herbs, laxatives & $6(18.7)$ \\
Other medications - antibiotics, emergency & \\
contraception & $2(6.2)$ \\
Other methods - smoked & $1(3.1)$
\end{tabular}

speaking and unemployed women, and that it was not associated with educational level or housing type (which was used as a proxy for socioeconomic status). This suggests that particular cultural beliefs, a reliance on self-medication for other problems, or even heightened stigma around abortion may be factors that provoke women to search for solutions to unwanted pregnancies outside the formal healthcare sector. Further in-depth interviewing and more quantitative data from larger samples are necessary to explore this.

The most common methods for self-induction in this population involved the use of remedies and herbal products rather than medications procured from backstreet providers. This is not dissimilar to methods used for self-induction in other countries. US women reported using misoprostol, self-medication with vitamin $\mathrm{C}$, aspirin or oral contraceptives, other foods and beverages, herbs, and in a small number of cases, physical manipulation. ${ }^{[13]}$ Jewkes et al. ${ }^{[6]}$ noted that in SA self-medication is a common first-line treatment response to health problems, but emphasised that even when the methods used are not necessarily harmful, these practices potentially have harmful consequences. While the failed attempts at self-induction in this sample of women did not appear to have been harmful to their health, they may have pushed women later into pregnancy and contributed to their seeking a second-trimester abortion, which is associated with an increased risk of complications. ${ }^{[14]}$

Use of physical trauma for self-induction after the legalisation of abortion services in SA had dramatically reduced by $2005^{[6]}$ and was absent in our sample. While misoprostol is potentially less hazardous than physical means, without knowledge of dose requirements and possible complications, using misoprostol supplied through backstreet sources remains a potentially hazardous path for women seeking assistance.

In SA, where abortion is legally permitted within broad criteria, access to safe abortion services remains suboptimal. A scarcity of nurse providers has become apparent in recent years, and those working in abortion facilities describe services as overcrowded and overburdened. The dearth of trained and willing abortion care providers has compromised access to safe abortion services. ${ }^{[8]}$ In the Western Cape, less than $75 \%$ of the 40 designated facilities are functional, and at some services are offered only every second week or on a monthly basis. First-trimester services are provided at 29 facilities, and second-trimester services at just 8 of these facilities (unpublished data, 2010). Where access is problematic, women are likely to resort to unsafe and clandestine abortion services. The current plethora of advertisements by illegal providers in public urban spaces, coupled with limited availability of services and service providers, is an alarming trend that could contribute to maternal mortality resulting from unsafe procedures.

\section{Study limitations and recommendations}

The data presented in this report are limited to women seeking secondtrimester abortion at public facilities. Our interpretations of prevalence and methods of self-induction are therefore not generalisable to women outside the formal healthcare system, those with incomplete abortions resulting from self-induction, or those presenting for abortion in the first trimester of an unwanted pregnancy.

As this study included only women in their second trimester, and the whole sample may therefore be considered somewhat delayed, associations between self-induction and delays in accessing services 
are less likely to be uncovered than in a broader-based sample in which women in both the first and second trimesters are included.

To address the issue of unsafe practices, further efforts should be channelled towards informing women in the community about the availability of free services in the public sector and educating them about the dangers of self-induction and unsafe providers. In this exploratory study we did not ask women why they chose self-induction before accessing legal services. Further details would have provided useful information to inform efforts to prevent unsafe practices. Service provision also needs to be strengthened by making services more acceptable and woman-friendly, including reducing the delays experienced by women seeking abortion care at public facilities. ${ }^{[9]}$

\section{Conclusion}

Seventeen per cent of women accessing second-trimester abortion services in the Western Cape public sector had resorted to selfinduction before entering legal public sector services. This reflects a high percentage of unsafe practices despite SA's liberal abortion law and the relatively widespread availability of abortion services in urban settings. No significant associations with self-induction were found in this exploratory study.

Funding. Funding for this research was provided by the Safe Abortion Action Fund.

\section{References}

1. Jewkes R, Rees H. Dramatic decline in abortion mortality due to the Choice on Termination of Pregnancy Act. S Afr Med J 2005;95(4):250.

. World Health Organization. Information Sheet: Safe and Unsafe Induced Abortion. Global and Regional Levels in 2008, and Trends during 1995 - 2008. Geneva: WHO, 2012.

3. Sedgh G, Singh S, Shah IH, Ahman E, Henshaw SK, Bankole A. Induced abortion: Incidence and trends worldwide from 1995 to 2008. Lancet 2012;379(9816):625-632. [http://dx.doi.org/10.1016/S01406736(11)61786-8]

4. Reddy SP, James S, Sewpaul R, et al. Umthente Uhlaba Usamila - The South African Youth Risk Behaviour Survey 2008. 2010. http://www.mrc.ac.za/healthpromotion/yrbs_2008_final_report.pdf (accessed 13 December 2013).

5. Grossman D, Holt K, Peña M, et al. Self-induction of abortion among women in the United States. Reprod Health Matters 2010;18(36):136-146. [http://dx.doi.org/10.1016/S0968-8080(10)36534-7]

6. Jewkes RK, Gumede T, Westaway MS, Dickson K, Brown H, Rees H. Why are women still aborting outside designated facilities in metropolitan South Africa? BJOG 2005;112(9):1236-1242. [http://dx.doi.org/10.1111/ j.1471-0528.2005.00697.x]

7. Orner PJ, de Bruyn M, Barbosa RM, Boonstra H, Gatsi-Mallet J, Cooper DD. Access to safe abortion: Building choices for women living with HIV and AIDS. J Int AIDS Soc 2011;14:54. [http://dx.doi.org/10.1186/17582652-14-54]

8. Harries J, Stinson K, Orner P. Health care provider's attitudes towards termination of pregnancy: A qualitative study in South Africa. BMC Public Health 2009;9:296. [http://dx.doi.org/10.1186/1471-2458-9-296]

9. Grossman D, Constant D, Lince N, Alblas M, Blanchard K, Harries J. Surgical and medical second trimester abortion in South Africa: A cross-sectional study. BMC Health Serv Res 2011;11:224. [http://dx.doi. org/10.1186/1472-6963-11-224]

10. Department of Health. Saving Mothers: Third Report on Confidential Enquiries into Maternal Deaths in South Africa 2002 - 2004. Pretoria: Department of Health, 2006.

11. Department of Health. Saving Mothers: Fourth Report on Confidential Enquiries into Maternal Deaths in

South Africa 2005- 2007. Pretoria: Department of Health, 2009.

2. Department of Health. Saving Mothers: Fifth Report on Confidential Enquiries into Maternal Deaths in South Africa 2008 - 2010. Pretoria: Department of Health, 2012.

13. Jones RK. How commonly do US abortion patients report attempts to self-induce? Am J Obstet Gynecol 2011;204(1):23.el-4. [http://dx.doi.org/10.1016/j.ajog.2010.08.019]

14. Bartlett LA, Berg CI, Shulman HB, et al. Risk factors for legal induced abortion-related mortality in the United Bartett LA, Berg CJ, Shulman HB, et al. Risk factors for legal induced abortion-related mortality in the
States. Obstet Gynecol 2004;103(4):729-737. [http://dx.doi.org/10.1097/01.AOG.0000116260.81570.60]

Accepted 17 October 2013 